\title{
A Case Report of Atrial Myxoma presenting with Cardioembolic Stroke and treated with Intravenous Thrombolytic Therapy
}

Ismail II ${ }^{*}$, Salama SS ${ }^{2}$ and Mansour OY ${ }^{3}$

${ }^{1}$ Neurology Specialist, Department of Neurology, Alexandria University

${ }^{2}$ Associate Lecturer, Department of Neurology, Alexandria University

${ }^{3}$ Associate Professor, Department of Neurology, Alexandria University

${ }^{*}$ Corresponding author: Ismail II, Neurology Specialist, Department of Neurology, Alexandria University, E-mail: dr.ismail.ibrahim2012@gmail.com

Citation: Ismail II, Salama SS, Mansour OY (2015) A Case Report of Atrial Myxoma presenting with Cardioembolic Stroke and treated with Intravenous Thrombolytic Therapy. J Case Rep Stud 3(2): 205. doi: $10.15744 / 2348-9820.2 .505$

Received Date: February 12, 2015 Accepted Date: April 07, 2015 Published Date: April 09, 2015

\begin{abstract}
Background: Cardiac myxomas are a potential source of emboli to the brain and elsewhere in the vascular tree. Myxomas are the most common benign primary cardiac tumor in adults. The commonest location of cardiac myxomas is in the left atrium followed by the right atrium and the remainder develops in the ventricles and rarely in the heart valves. Embolic events occur in approximately $40-50 \%$ of patients with myxomas. Crebrovascular strokes secondary to myxomas account for less than $1 \%$ of all ischemic strokes but should be considered in the differential diagnosis of patients with stroke in young.

Case Description: We report a case of a 42 year-old female patient with a recently discovered left atrial myxoma presenting with sudden onset of ischemic cardioembolic stroke of the right middle cerebral artery. She presented within the therapeutic window of intravenous thrombolytic therapy and was successfully treated.

Conclusion: Atrial myxomas are the most common benign primary cardiac tumors in adults and can present in several ways. Embolic ischemic stroke is the most common neurological complication and should be suspected in cases of stroke in young especially in the absence of conventional risk factors. Systemic thrombolysis seems to be safe and effective in treating acute stroke secondary to atrial myxomas especially in young patients and in patients treated early.
\end{abstract}

Keywords: Myxoma; Cardioembolic; Stroke; Intravenous Thrombolysis

\section{Introduction}

Atrial myxomas are the commonest cardiac tumors. Despite being benign, embolic phenomenon occurs in approximately 40-50 $\%$ of cases either to the brain or elsewhere in the vascular tree. The embolic source may be composed of tumor tissue itself, blood clot or both [1].

Myxomas are a rare cause of ischemic stroke accounting for less than $1 \%$ of all stroke cases. However, they should be considered in the differential diagnosis of cases with stroke in young in the absence of conventional stroke risk factors. Some case reports of ischemic stroke secondary to emoli originating from atrial myxomas had been successfully treated with IV thromolytic therpy and it seems to be safe and effective in such cases [2].

We report a case of a 42 year-old female patient with recently discovered left atrial myxoma and presenting with sudden onset of ischemic cardioembolic stroke of the right middle cerebral artery within the therapeutic window of intravenous thrombolytic therapy and successfully treated.

\section{Case Report}

A 42 year-old female patient with no history of Diabetes Mellitus or Hypertension presented to the Emergency Room with a recent history of sudden onset left sided hemiparesis, left upper motor neuron facial palsy and dysarthria 2.5 hours prior to the hospital arrival.

On arrival the patient was vitally stable; afebrile, blood pressure was $140 / 85$, heart rate was 77 beat per minute with normal rhythm and heart sounds and respiratory rate was 22 cycle per minute.

Neurological assessment showed a semi-conscious patient, orientated to place and person but not to time and hardly obeying commands. Language assessment showed normal comprehension but with moderate dysarthria. Cranial nerves examination showed left UMN facial palsy. Muscle power was of MRC Grade (0-1) in the left side and normal on the other side. Deep tendon jerks were more brisk on the affected side with an extensor plantar response on the left side. National Institutes of Health Stroke Scale (NIHSS) on presentation was 21. 
Past medical history of the patient showed a recently discovered large left atrial myxoma that presented with cough, palpitation and chest tightness one month before the onset of stroke and the patient was assigned for surgical excision unfortunately the same day the stroke occurred. Her Echocardiogram showed a large $(8 \times 6 \times 7 \mathrm{~cm})$ left atrial mass with areas of degeneration and mildly impairing flow through mitral valve (Figure 1). Other Echocardiogram parameters were within normal limits with no intracardiac thrombi and EF was 65\%. She had no history of previous TIAs.

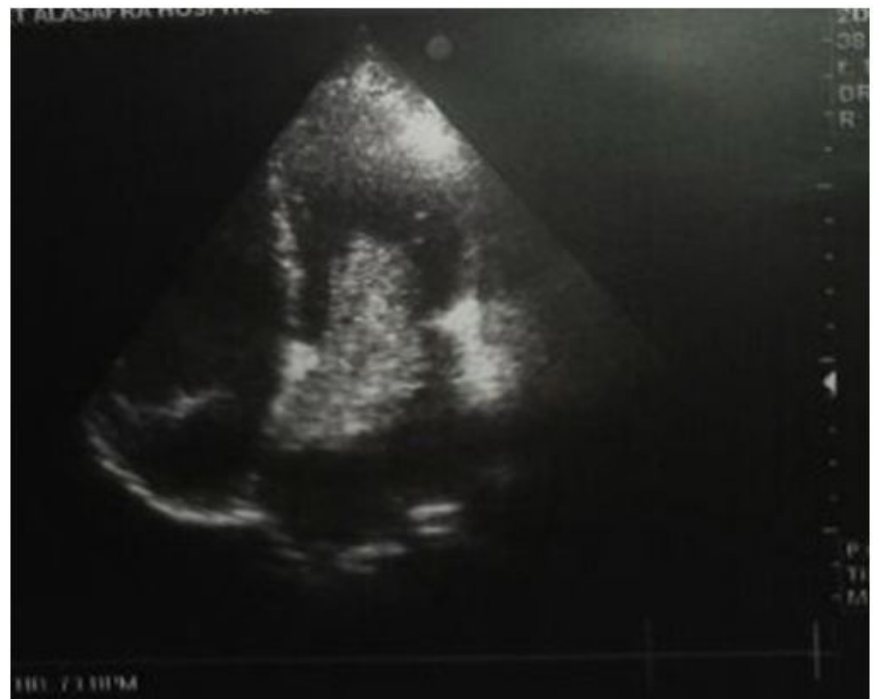

Figure 1: Echocardiogram showing large oval left atrial myxoma attached to fossa ovalis and projecting into left ventricle

Initial NECT showed Early Ischemic Signs (EIS); slight blurring of the border between the gray and white matter, with sulcal effacement in the right frontotemporal lobe with a Hyperdense MCA sign (Figure 2).

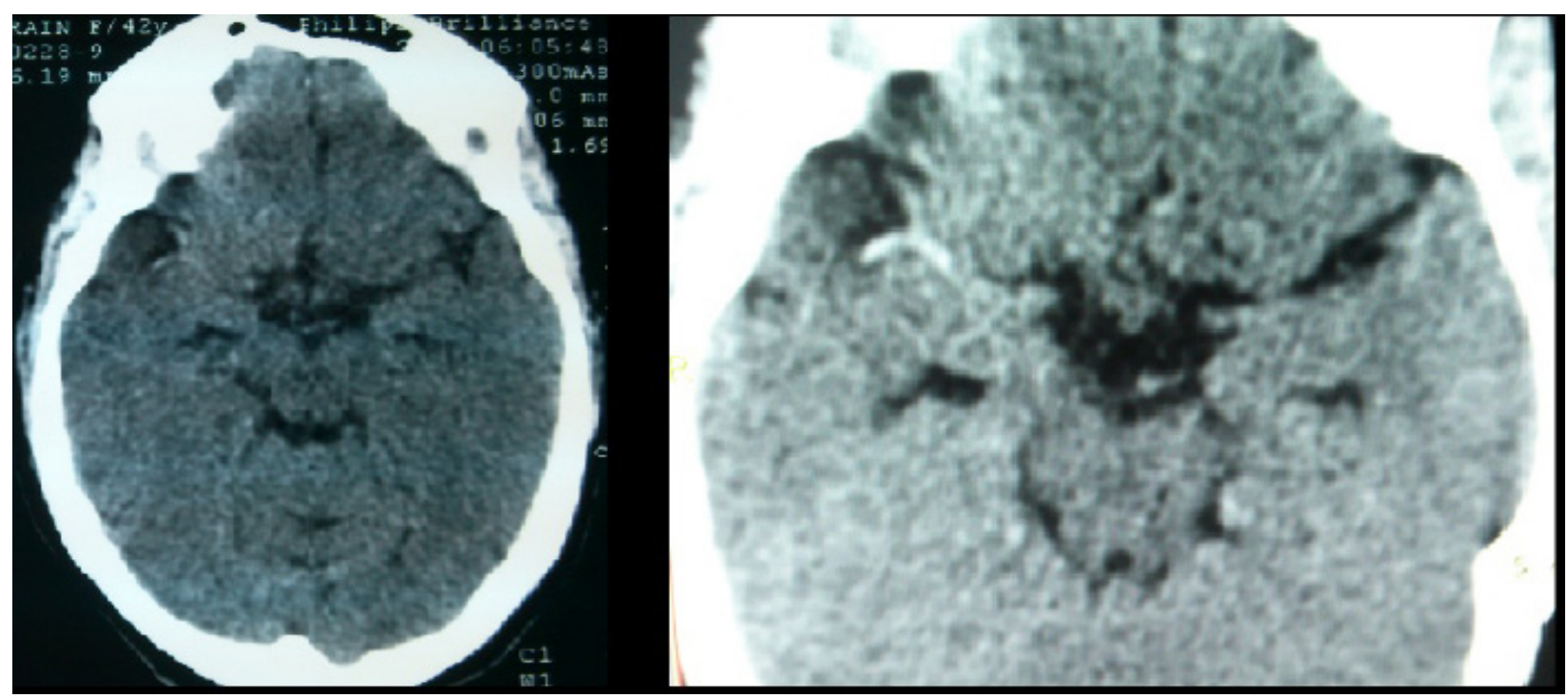

Figure 2: NECT showing "Hyperdense MCA Sign" on the right side

The patient received intravenous recombinant tissue plasminogen activator (IV rtPA) 3.5 hours after the onset of the stroke with a dose of $(0.9 \mathrm{mg} / \mathrm{kg})$ over one hour. The patient started to show improvement of the clinical condition within 20 minutes of treatment in the form of improved alertness, motor power and speech. Follow up NIHSS was 15 after 24 hours.

CT Angiography was done after treatment and showed recruitment of the leptomeningeal collaterals despite incomplete recanalization of MCA (Figure 3).

Follow up NECT after 24 hours showed hypodensity involving right MCA territory but with no hemorrhagic transformation (Figure 4).

Patient's condition was stable during the hospital stay and anticoagulation was initiated after 2 weeks to keep INR between 2.0 -3.0. Surgery was contraindicated at the time of admission. Our patient started Physical Therapy and rehabilitation and condition showed further mild improvement 1 month after stroke with NIHSS of 13. 


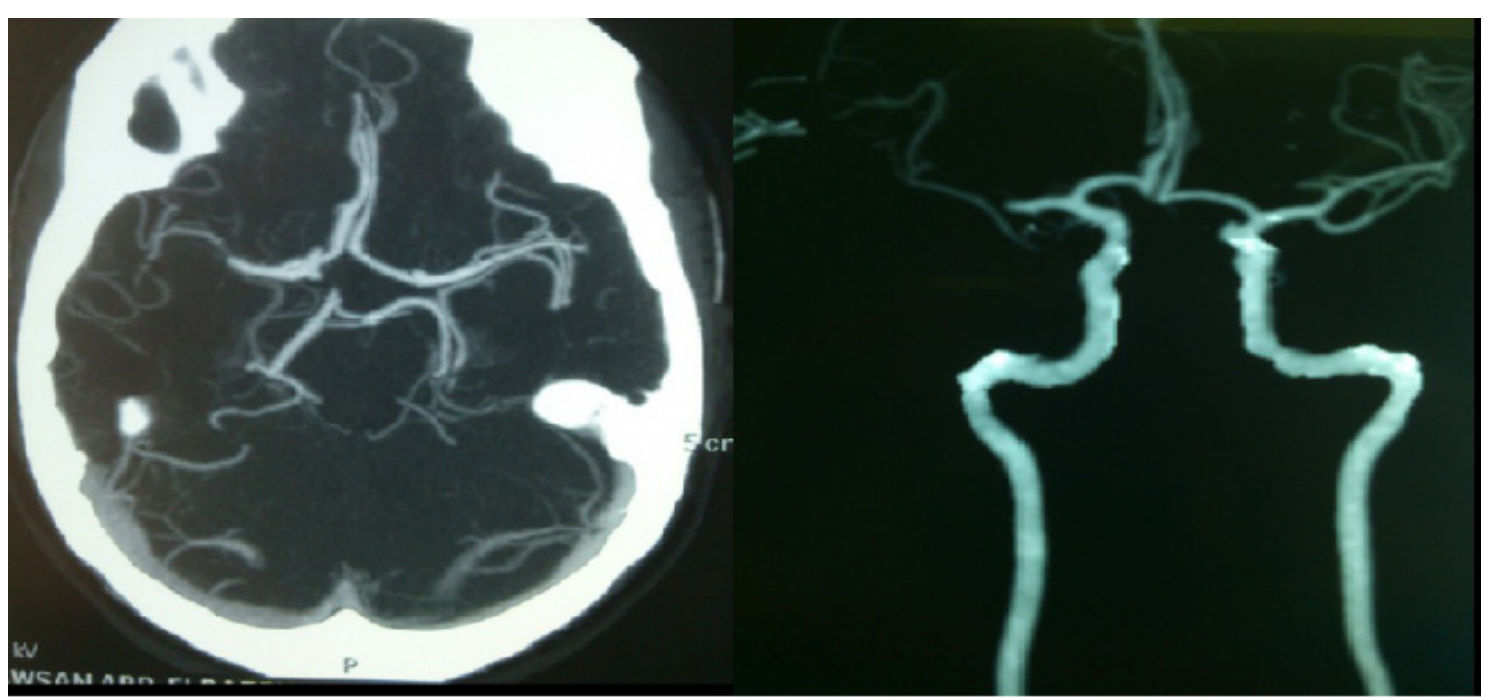

Figure 3: CTA showing occlusion of right MCA with recruitment of pial collaterals

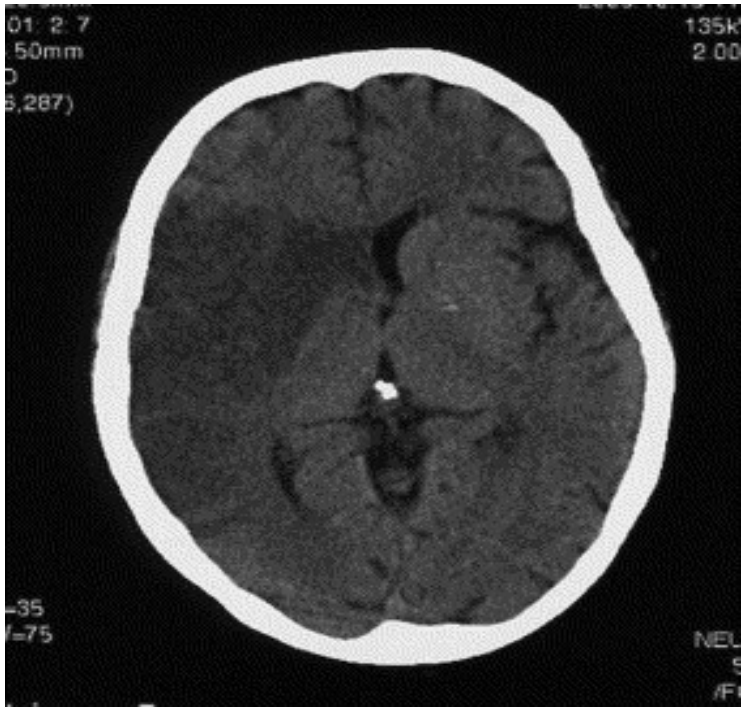

Figure 4: Follow up NECT showing hypodensity involving right MCA territory with no hemorrhagic transformation

\section{Discussion}

Primary tumors of heart are rare, with an incidence between (0.0017\% to $0.19 \%)$ in unselected patients at autopsy [3]. Atrial myxoma is the most common benign cardiac tumor in young adults. They can present in all age groups but they are particularly frequent between third and six decades of life. The commonest location of cardiac myxomas is in the left atrium followed by the right atrium and the remainder develops in the ventricles and rarely in the heart valves. They are sporadic in most of the cases but at least $7 \%$ are familial [4].

The presentation of atrial myxoma often comprises a diagnostic triad; (a) symptoms due to obstruction of cardiac outflow, (b) constitutional symptoms or (c) symptoms due to embolism. Neurological complications are common in patients with myxoma, occurring in approximately one third to half of these patients. Embolism occurs in $40-50 \%$ of the patients with myxomas. Ischemic stroke is the most frequent embolic manifestation and should be considered for differential diagnosis of stroke in young though it is a rare cause [5].

Cerebrovascular strokes secondary to myxomas account for less than $1 \%$ of all ischemic strokes. It is found more in young patients who present with ischemic stroke ( 1 in 250) than older patients ( 1 in 750). Stroke is the initial presentation in 50\% of cases of atrial myxoma, and in $75 \%$ of the cases it is seen with left atrial myxoma. It is more common in females than in males [6]. The mechanism for embolic stroke in atrial myxoma is either by fragment from the tumor itself or from a thrombus developed on the surface of tumor [2].

Cerebrovascular strokes secondary to myxomas account for less than $1 \%$ of all ischemic strokes. It is found more in young patients who present with ischemic stroke ( 1 in 250) than older patients ( 1 in 750). Stroke is the initial presentation in 50\% of cases of atrial myxoma, and in $75 \%$ of the cases it is seen with left atrial myxoma. It is more common in females than in males [6]. The mechanism for embolic stroke in atrial myxoma is either by fragment from the tumor itself or from a thrombus developed on the surface of tumor [2]. 
In our case, the ischemic stroke was most probably due to fragments of the tumor itself rather than a thrombus as the patient's Echocardiogram done 1 week before the stroke did not show any thrombi and a follow up transthoracic Echo was done later and also did not show thrombus formation.

The role of thrombolysis in acute ischemic stroke in patients with atrial myxoma is not well established. There are few data available regarding its safety and efficacy in such situation in some case reports and it showed mixed results. Partial success was observed using intra-arterial local thrombolysis but with higher risk of hemorrhage. Intravenous recombinant tissue plasminogen activator showed favorable outcome with less hemorrhagic transformation in some case reports [7]. Mechanical thrombectomy was found to be a very reasonable choice for such patients especially when the embolus is composed mainly of tumor tissue, but it would be difficult to speculate that the patient is having myxoma if he was not previously diagnosed [8]. Fortunately our patient showed improvement with no hemorrhagic transformation. The favorable outcome might be due to the young age of the patient, absence of conventional risk factors, relatively early administration of rtPA and absence of associated microaneurysms in angiography. Contrary to some expert opinions, systemic thrombolytic therapy may be safely and effectively used to treat acute ischemic strokes from atrial myxoma especially in young patients with short door-to-needle time $[9,10]$. Further research in a larger population is necessary to determine the best effective and safe therapy for infarction associated with cardiac myxomas $[11,12]$.

\section{Conclusion}

Atrial myxomas are the most common benign primary cardiac tumors in adults and can present in several ways. Embolic ischemic stroke is the most common neurological complication and should be suspected in cases of stroke in young especially in the absence of conventional risk factors. Systemic thrombolysis seems to be safe and effective in treating acute stroke secondary to atrial myxomas especially in young patients and in patients treated early.

\section{References}

1. Ibrahim M, Iliescu C, Safi HJ, Buja ML, McPherson DD, et al. (2008) Biatrial myxoma and cerebral ischemia successfully treated with intravenous thrombolytic therapy and surgical resection. Tex Heart Inst J 35: 193-5.

2. Nishimura H, Nakajima T, Ukita T, Tsuji M, Miyake H, et al. (2010) A case of acute cerebral infarction associated with left atrial myxoma treated by intravenous tissue plasminogen activator. Jpn J Stroke 32: 156-62.

3. Reynen K (1995) Cardiac myxomas. N Engl J Med 333: 1610-7.

4. Hart RG, Albers GW, Koudstaal PJ (1998) Cardioembolic stroke. Cerebrovascular disease: pathophysiology, diagnosis and management. London: Blackwell Science 1392-429.

5. Pinede L, Duhaut P, Loire R (2001) Clinical presentation of left atrial myxoma. A series of 112 consecutive cases. Medicine (Baltimore) 80: 159-72.

6. Hatayama S, Ogata T, Okawa M, Higashi T, Inoue T, et al. (2012) Ischemic stroke induced by a left atrial myxoma. Brain Nerve 64: 1175-9.

7. Sun MC, Tai HC, Lee CH (2011) Intravenous thrombolysis for embolic stroke due to cardiac myxoma. Case Rep Neurol 3: 21-6.

8. Gobin YP, Starkman S, Duckwiler GR, Grobelny T, Kidwell CS, et al. (2004) MERCI 1: a phase 1 study of Mechanical Embolus Removal in Cerebral Ischemia. Stroke 35: 2848-54.

9. Kulkarni GB, Yadav R, Mustare V, Modi S (2014) Intravenous thrombolysis in a patient with left atrial myxoma with acute ischemic stroke. Ann Indian Acad Neurol 17: 455-8.

10. Kohno N, Kawakami Y, Hamada C, Toyoda G, Bokura H, et al. (2012) Cerebral Embolism Associated with Left Atrial Myxoma That Was Treated with Thrombolytic Therapy. Case Reports in Neurology 4: 38-42.

11. Nagy CD, Levy M, Mulhearn TJ, Shapland M, Sun H, et al. (2009) Safe and effective intravenous thrombolysis for acute ischemic stroke caused by left atrial myxoma. J Stroke Cerebrovasc Dis 18: 398-402.

12. Acampa M, Tassi R, Guideri F, Marotta G, Monti L, et al. (2011) Safety of intravenous thrombolysis in ischemic stroke caused by left atrial myxoma. Curr Drug Saf 6: 343-5.

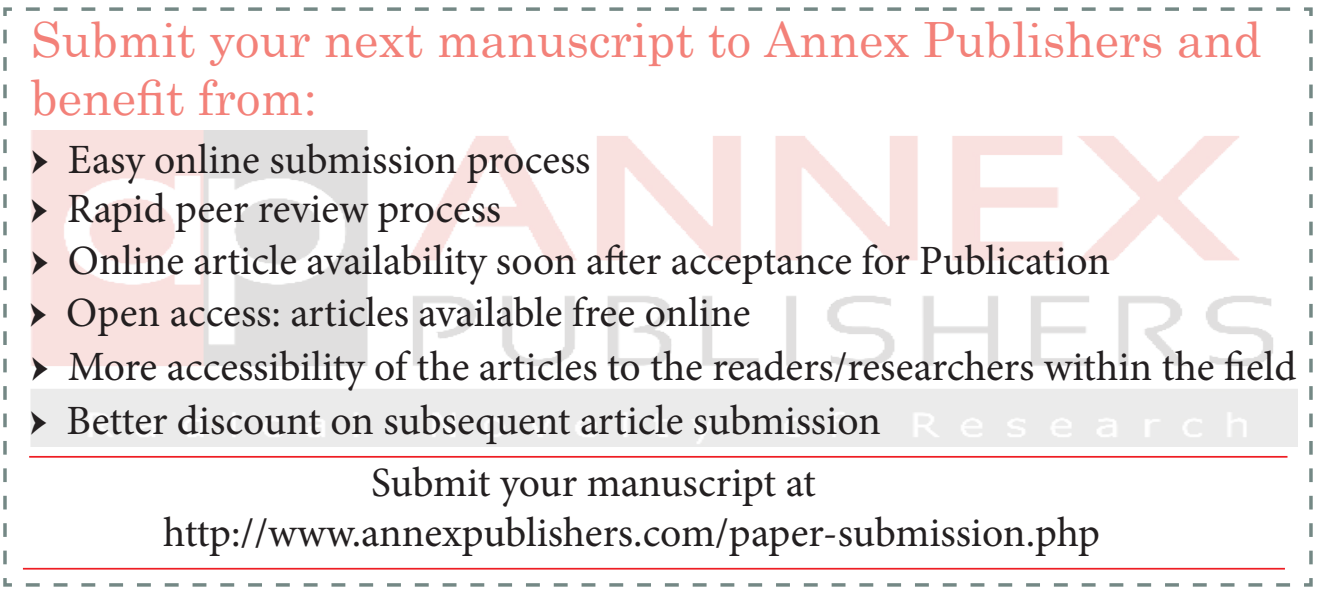

